\title{
Experience and Design Recommendations for Picture Archiving and Communication Systems in the Surgical Setting
}

\author{
Stephen M. Pomerantz, Eliot L. Siegel, Zenon Protopapas, Bruce I. Reiner, and Elliott R. Pickar
}

\begin{abstract}
An analysis of the efficacy of a picture archiving and communication system (PACS) in the surgical domain was undertaken at the Baltimore Veterans Affairs Medical Center. Interviews with surgeons and staff were conducted and supplemented by direct radiologist observation in the operating room (OR) and surgical outpatient clinic to determine patterns of routine clinical PACS use, levels of satisfaction both within and outside of the OR, and perceptions of the relative efficacy of the system in comparison to film. These data as well as suggestions from the surgical staff members were used to make recommendations for specific modifications in PACS design and operation to improve the current system and to help prescribe design improvements for future PAC systems. A high level of satisfaction with the system was found and the use of PACS was favored over film by a majority of surgeons and their staff. Findings of this study suggest that the design of a hospital-wide PAC system must have the flexibility to accommodate the specific requirements of a wide variety of end-users in their unique hospital environments.
\end{abstract}

Copyright $\odot 1996$ by W.B. Saunders Company

KEY WORDS: picture archiving and communication system (PACS), filmless hospital, surgery, operating room (OR), design, radiology, imaging.

$\mathbf{H}$ OSPITAL-WIDE implementation of picture archiving and communication systems (PACS) promises clinicians unparalleled access to diagnostic imaging. ${ }^{1,2}$ However, a filmless environment presents formidable challenges in accommodating the specific and widely varying needs of different medical specialties and their special environments. This report presents updated preliminary data from an ongoing examination ${ }^{3}$ of the use of PACS by surgeons and their staff in the operating rooms and surgical outpatient clinics at the Baltimore Veterans Affairs Medical Center (BVAMC). The analysis was performed to provide assistance in improving the PACS and to suggest ways to optimize future PACS design. This assessment and re-engineering is expected to serve as a model for tailoring a hospital-wide PACS to other unique medical center environments such as the emergency room (ER), intensive care unit (ICU), and patient care wards.

\section{Background}

The use of a hospital wide PACS (Loral Medical Imaging Systems, Chicago, IL) has enabled "filmless operation" at the BVAMC, a 300 bed teaching hospital located adjacent to the University of Maryland Medical Center (Baltimore), for approximately $2 \frac{1}{2}$ years. ${ }^{4-6}$ The 42 PACS workstations use a Macintosh (Apple Computer, Cupertino, CA) IIFX or Quadra 950 system (Apple Computer). These are distributed throughout the BVAMC including the diagnostic imaging department, patient-care wards, conference rooms, surgical and medical outpatient clinics, ICUs, ER, and the operating room (OR). Workstations are directly connected via fiberoptic cable to a central server using a proprietary network protocol that enables rapid imaging display. Images are initially stored on-line for approximately 2 to 3 weeks after acquisition on a central "redundant array of inexpensive disks" (RAID), a high performance image file server with a several second access time. Studies are then archived to an optical jukebox where retrieval times generally range from 1 to 5 minutes (occasionally longer for a complex study or when many studies are queued). Once images are retrieved from the jukebox, they remain "on-line" on the RAID server for a period of time determined by the date and time of their last access. When imaging studies are performed, previous examinations of the same modality are automatically "fetched" from the optical jukebox.

The BVAMC's six functional general purpose operating rooms are each equipped with a

From the Department of Diagnostic Imaging and Nuclear Medicine, Baltimore Veterans Affairs Medical Center, and the Department of Diagnostic Radiology, University of Maryland Medical System, Baltimore, $M D$.

Address reprint requests to Stephen M. Pomerantz, MD, Department of Diagnostic Radiology, University of Maryland Medical System, 22 S Greene St, Baltimore, MD 21201.

Copyright 101996 by W.B. Saunders Company

0897-1889/96/0903-0005\$3.00/0 
two-monitor $(1,152 \times 1,078$ pixels $)$ workstation (Fig 1). Two additional cystography ORs share a seventh workstation. Image manipulation tools on these workstations are identical to those available in the radiology department. The workstations display both images and radiology reports. A separate, proprietary hospital information system (HIS)/radiology information system (RIS) PACS available throughout the hospital is present in each OR. ${ }^{4}$ This system displays and stores patient information and clinical images such as digitized photographs, endoscopy, bronchoscopy, and laparoscopic images. Radiology images are stored (at lower resolution than the radiology $\mathrm{PACS}$ ) on this second $\mathrm{PACS}$, which serves as a backup in case of failure of the radiology $\mathrm{PACS}$.

The surgical outpatient clinic consists of eight examination rooms with a central "team room" containing a two-monitor $(1,152 \times 1,078$ pixels $)$ workstation as well as light boxes for outside films.

\section{MATERIALS AND METHODS}

Day to day experiences, lessons learned, and suggestions for improvement were gleaned from interviews with approximately 30 members of the surgical staff including surgeons representative of the different surgical subspecialties, physician assistants, nurses, and technicians. Additionally, operation of PACS by surgeons and staff was observed directly by experienced PACS radiologists in the OR and surgical outpatient clinic. Areas assessed included: patterns of image review, availability of PACS images and radiology reports, quality of PACS images, PACS workstation design and maintenance, patterns of OR workstation usage, intraoperative imaging, PACS use in the outpatient clinic,

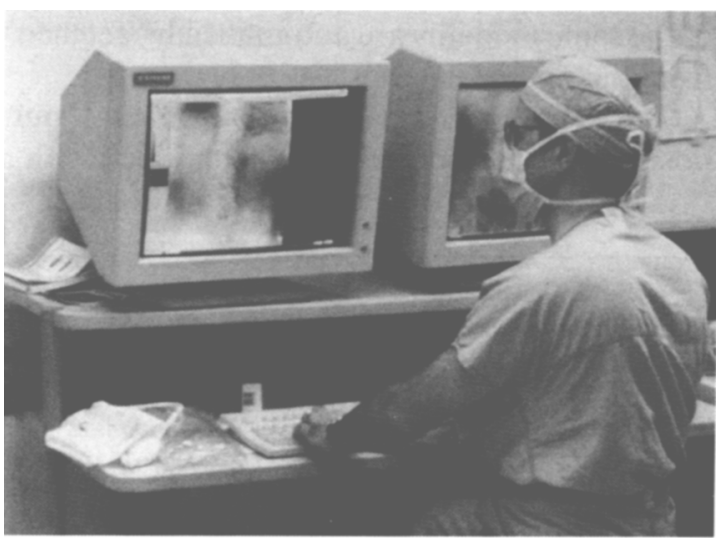

Fig 1. BAVMC surgeon operates a two-monitor (1,152 $\times 1,078$ pixels) Macintosh Quadra 950 workstation (Apple Computer) in the operating room. potential for three-dimensional imaging, adequacy and suitability of PACS training, and overall acceptance of PACS.

All BVAMC surgeons also have privileges at the University of Maryland Hospital where imaging is film-based. Their experiences in this traditional environment served as a standard of comparison for the evaluation of PACS.

\section{RESULTS}

\section{Patterns of Image Review}

Image review by surgeons primarily occurs outside of the OR where imaging is used for diagnosis and to determine the site, scope, and approach of the surgical procedure. Images are often re-reviewed in the OR just before surgery. Overall, the PACS is only used $15 \%$ to $20 \%$ of the time during surgery as estimated by surgeons and staff although figure varies substantially among surgical specialties. Use of images varies from ophthalmologists who report that they do not use the PACS during surgery at all to the general surgeons and otorhinolaryngologists (Ear, Nose, and Throat surgeons) who use the PACS in less than $15 \%$ of cases, to orthopedic and neurosurgeons who use PACS during surgery in more than $90 \%$ of cases. PACS is used intraoperatively either to display images as a "roadmap" to guide the procedure or for display of intraoperative imaging studies such as cholangiography, cystography, prosthesis/hardware positioning, or stereotactic brain biopsies. Orthopedic, vascular, and neurosurgeons more frequently use images as a roadmap whereas general surgeons rarely do.

Although in general, surgeons do not display preoperative PACS images during surgery, they consider their availability for potential use essential. Interestingly, surgeons admit that in a film environment, radiographs are often hung as "window dressing, more for ambiance than utility." However, in a PACS-based OR they are often content not to display such images, confident that they would be available if needed.

\section{Availability of PACS Images and Radiology Reports}

Image availability was uniformly cited as the most valuable benefit of PACS when compared to film. The system allows surgeons and staff to avoid the common problems of film loss and time-consuming retrieval from the film library. Operating room logs report no cases postponed 
or cancelled because of lack of image availability. However, satisfaction with image availability is tempered by occasional long wait times when older studies have to be retrieved from the optical disk to the RAID (on-line) server. Solutions offered include a larger on-line capacity, faster hardware/software, automatic image retrieval for any patient on the OR schedule, and the ability of OR personnel to initiate an emergency fetch to prioritize critical images in the optical disk jukebox retrieval queue.

As experience with the PACS has grown, surgeons accustomed to rapid image access are more demanding in regard to rapid report access. The official radiology interpretation text is available on the workstation at the time of image display. This feature, considered by the surgeons to be extremely beneficial, has obviated the time consuming tasks of looking up results in the chart or on other computer systems. It also has decreased the number of trips to the radiology department for interpretations (resulting in less interaction with radiologists). In the case of very recent studies where reports may not yet have been dictated or transcribed, an on-line interpretation is frequently not available. As a result, surgeons have requested more expeditious reporting. They have also suggested a preliminary audio report be available on the PACS workstation almost as soon as the imaging study is performed. Integration of a digital voice server into the $\operatorname{PACS}^{7,8}$ would allow a voice file containing a preliminary report and later the official dictation (allowing it to be available before being transcribed) to be associated with the image files. Conversion of the audio file to text via speech recognition software would be eliminate the transcription step altogether. ${ }^{1}$

\section{PACS Image Quality}

Overall image quality on PACS is judged at least equivalent to film by all surgeons. Most rate PACS image quality better than film when taking into account the ability to modify contrast using the window/level tool. The exception to this general preference for soft copy is magnetic resonance imaging (MRI). Most surgeons report MRI images to be of lesser quality than film. Radiologists observing the surgeons have concluded that the most likely explanation is the particular difficulty surgeons have obtaining satisfactory window and level settings on each of the multiple sequences typically obtained on an MRI examination.

\section{PACS Workstation Design}

Location. Satisfaction with positioning of the workstation controls and monitors has been variable depending on the particular room layout and workstation location. Most surgeons and staff surveyed believe the workstation components could be arranged in a more ergonomic fashion. Issues raised include the distance from the OR table, height of the monitors, and inconvenient placement of keyboard and pointing device with respect to the OR table. Nurses in particular are concerned that the workstation obstructs OR traffic flow in some of the rooms. Suggestions include installing the monitors on ceiling-mounted articulated arms to allow variable positioning including the ability to bring the monitors to eye-level close to the OR table. Use of a wireless keyboard/pointing device (with a sterile covering) located at the OR table and/or a foot pedal were suggested.

Complexity of controls. Surgical staffs have found workstation controls to be complicated and have difficulty using many of the workstation functions. Although many feel that this is a result of the need for more training, there is a unanimous desire for less complex, more automated controls. There is particular difficulty obtaining satisfactory window and level contrast settings. Window/Level controls have been found to be nonintuitive. As judged by radiologist observers, images displayed on OR monitors are often displayed using suboptimal window/level settings. All surgeons and staff surveyed are in agreement that default modes for automatic image display ${ }^{9}$ would be of value. Window/level settings "presets" (available on workstations within the radiology department) are desired.

Ambient light. Image conspicuity is decreased by ambient light. ${ }^{10,11}$ The ability to dim the lights with a foot pedal and nonglare monitors has been suggested.

Screen saver. When first installed, all hospital workstation monitors were designed to go blank after 5 minutes of nonuse. Initially the surgeons and staff interpreted this as a worksta- 
tion malfunction. Frantic calls from the OR to the radiology department were not infrequent during early weeks of use. The "screen savers" in the OR have consequently been reset with a longer delay and the default workstation "inactivity" timer has been reset to 8 hours.

Manipulation of multiple studies/sequences. The surgeons report difficulty using the workstation software to compare images from multiple studies or from studies with multiple parts such as enhanced and unenhanced CT examinations. Viewing MRI examinations is described as particularly cumbersome because of the inability to satisfactorily compare images from the multiple sequences. This is due to the inability of the current software to separate multiple sequences or portions of a study. Consequently, the surgeons usually ask that MRI studies be printed onto film before using them in the OR. More sophisticated image navigation software was requested. Most surgeons believe that the use of additional monitors would not be helpful because they would contribute to increased clutter in the OR and clinic.

Lack of true 1:1 aspect ratio. Orthopedic surgeons use prosthesis templates, which they physically place over the image on the screen. This requires the image to be formatted $1: 1$ as would be the case with film. The surgeons have found that the workstation setting labeled as 1:1 does not actually produce an image of equivalent size to a film image and consequently they adjust the image manually with the magnification controls. They have requested that the software be modified appropriately.

Inability to superimpose images. Orthopedic surgeons often superimpose pre- and postprocedure films to assess adequacy of intervention. For example, in the case of a fracture, a prereduction film is often superimposed over the postreduction image to assess for change. Currently this superimposition of images is not supported by the workstation software.

\section{Workstation Operation and Maintenance}

Patterns of PACS workstation operation in the $O R$. The degree to which the surgeon operates the workstation personally or delegates this task to OR staff varies widely. Often the surgeon will display and arrange images just before surgery and have a nurse use the workstation during the surgery, usually to page through already arranged and formatted images. Frequently one of the assistant surgeons will "break scrub" to operate the workstation. Alternatively, the primary surgeon will often don a second pair of gloves and operate the workstation him or herself. The gloves are then removed before resuming surgery. The magnification, window/level, and measurement tools are the most frequent image manipulation tools used. Angle measurement capabilities are frequently used by orthopedic surgeons.

Maintenance issues. Workstations are described as "dust collectors" by many surgical staff members. Regular OR and clinic custodial staff have tended to avoid cleaning the PACS components for fear of damaging the equipment. The current routine cleaning and maintenance by radiology maintenance staff was not considered adequate. Although not yet reported as a problem by PACS maintenance staff, there is also a concern that powder from latex gloves might damage the workstation keyboard. If this becomes a significant problem, placing a plastic cover over the keyboard may be of value.

Fear of system malfunction. Although no surgeons recall ever postponing or cancelling a procedure because of a PACS malfunction, there is concern over the possibility of a system failure with resulting inability to view images. Safeguards to minimize the likelihood of malfunction have been requested and measures to provide system redundancy are felt to be essential. The proprietary HIS/RIS PACS is available in all of the ORs containing all diagnostic images (at lower resolution) and serving as a backup for the commercial PACS. In-house PACS repair and troubleshooting staff members are on-call for problems that occur anywhere in the hospital during regular working hours; however, surgeons have reported occasional difficulty obtaining timely assistance. The lack of an in-house maintenance engineer for the PACS at night and on weekends is a potential problem. Given the potential for a system failure with loss of ability to retrieve and display images, the ability to print film as a backup is considered critical.

\section{Intraoperative Examinations}

Portable $x$-ray machines. Most intraoperative images are acquired using a portable $\mathrm{x}$-ray 
machine. The imaging plate is then delivered by the technologist to either the PACS image reader in the radiology department on the first floor or to the reader located one floor below the OR. Surgeons and staff perceive that there is a longer period between OR image acquisition and availability for review when compared to a film-based OR. Several explanations have been offered for this delay: (1) the lack of a film reader adjacent to the operating rooms. Typically in a film-based hospital, a film processor is located near the OR. Currently, the closest computed radiography plate reader is one floor below the operating room. (2) The inability to easily determine when images are ready for review. On-screen exam lists must be repeatedly checked to determine when images are ready for review. (3) Nonintuitive exam labeling and location within the system. Suggestions for improvement included placing a film reader near the OR. The workstation could feature an audible or visible indicator when images are ready. Also, automatic image display was suggested. A policy that would require the technologist to return to the OR and display the images was suggested as an incentive for the technologists to process images quickly. This would have the added benefit using the technologists' expertise in image retrieval and display and would free surgical staff from performing these tasks.

A C-arm fluoroscope is used for a small subset of portable OR studies. Currently an interface with the PACS is not offered by the vendor. Although the HIS/RIS PACS can capture video fluoroscopy images, these images have not been considered important for storage by surgeons.

Intraoperative image quality. Overall, the number of repeat intraoperative-operative images required is less with the PACS when compared to film. However, several surgeons perceive an increased number of patient positioning errors. They consider this a result of less attention to detail by radiology technologists who might believe (erroneously) that the surgeons can use image manipulation tools to correct any deficiency caused by suboptimal technique.

\section{PACS Use in the Surgical Outpatient Clinic}

The majority of image-based management decisions are made in the surgical clinic and efficient PACS operation in that area is deemed essential.

Workstation availability. A limited number of surgeons and staff alternate between multiple examination rooms, often at a somewhat "hectic" pace. Like most of the individual outpatient clinics, the surgical clinics are served by only a single PACS workstation. Consequently delays due to waiting for time for use of the workstation are not infrequent. Additional clinic workstations are unanimously desired by surgeons and staff. Surgical staff often report having to go into adjacent medical clinics to obtain access to additional workstations.

Image retrieval delay. Images previously archived to optical disk are not immediately available unless they were transferred from the jukebox to the RAID server before clinic hours. Requests for new examinations automatically result in the retrieval of prior studies from the jukebox. However, images from previous visits in patients not having a new study are not automatically retrieved. This task is often assigned to a resident, surgical technologist, or nurse. Clearly, this is not helpful for emergency or walk-in patients. Occasionally, repeat radiology exams have been performed that might have been avoided if the image was immediately available in short-term memory on the server. There is a strong desire for a system of automatic "prefetch" where image files retrieval from the long-term archive would be triggered by a patient scheduling an appointment or being registered for clinic.

Outside examinations. Frequently, radiographs from outside institutions are brought to the clinic. Surgeons find it difficult to compare images on a light box and images on the PACS monitor because images could not be displayed side by side. Time factors often prevent surgeons from having outside images digitized into the PACS system.

\section{Potential for Three-Dimensional (3D) Imaging}

Surgeons, to a much greater extent than radiologists, are accustomed to a $3 \mathrm{D}$ perspective. The PACS workstations can provide them with this perspective through the use of their ability to display sequential images rapidly (cine mode). Three dimensionally reconstructed images from computed tomography (CT) or MRI studies can be displayed on the workstation in 
the form of rotating images. Although only a small percentage of studies are reconstructed and displayed in this manner, the feedback from surgeons has been very favorable. An additional advantage of the cine mode is the ability to display angiograms, ultrasound studies, nuclear cinecardiographs, and magnetic resonance angiograms in "motion" to facilitate interpretation. These images can be displayed on the workstations in the operating rooms.

\section{PACS Training}

Formal PACS training is offered on a one-toone basis by appointment during working hours. Training was initially offered to physicians and physician assistants only. Only a small minority of the surgeons have taken advantage of the formal training stating that time limitations and scheduling conflicts prevented training session attendance. PACS workstation operation is consequently learned from colleagues or is "selftaught." All physician assistants surveyed took the training and report that it was valuable. The initial lack of nursing staff training was felt to be particularly detrimental in the OR because surgeons frequently call on the circulating nurses to operate the workstation. Training of nurses was implemented after the need was recognized. All nurses interviewed completed the tutorial. Despite the training, all nurses interviewed rated their PACS workstation skills as Iess than optimal. A common frustration was a situation where a nurse would struggle with a workstation function with an impatient surgeon looking over his or her shoulder.

Solutions offered for training issues include making training sessions more convenient and accessible. Although computer based training modules have been introduced to assist in this regard, the surgeons and staff indicate they have not been aware of them. They suggest these modules be available on all PACS workstation rather than on the training computers in the radiology department. There is consensus that training should be site-specific with more indepth teaching of functions typically used in the $O R$. Training personnel could visit the $O R$ on a regular basis to provide guidance on-site and answer questions as they come up. Certain OR personnel could be more highly trained and act as a resource for other staff. Making operation of the workstation less complex would in turn make training easier.

\section{Miscellaneous Issues}

Radiologist consultation. Because of the availability of imaging studies and interpretations in the surgeons own work areas, most surgeons describe spending less time in the radiology department resulting in less radiologist consultation. Surgeons suggested regular radiologysurgical conferences or radiologist attendance at surgical work rounds to increase consultation.

Security. System security is essential to prevent tampering with the PACS software and data and to protect patient medical record confidentiality. ${ }^{12}$ Although all appropriate staff are required to have a unique password for PACS access, none of those surveyed routinely use their own password to log onto PACS. In fact, a single password has been used by almost all of the surgical staff. This could be addressed by having the system periodically force the user to change passwords or by having the system deny access when the sign-on codes are already in use. Surgeons report difficulty keeping track of the many passwords a staff member has for the different computer systems at the BVAMC and the University of Maryland Hospital. A unified password for all systems including PACS has been suggested.

Role for film in a filmless hospital. Although the BVAMC is essentially a "filmless" hospital, film is still used in several circumstances: (1) Outside studies; surgeons are encouraged to have outside films digitized onto the PACS. (2) "Long cassette films" șuch as images of the complete spine or lower extremities. (3) MRI studies are often filmed because of the difficulties manipulating large numbers of images and sequences.

\section{Overall Acceptance of PACS}

PACS has been generally well accepted by the surgeons and staff at the BVAMC. None of those surveyed would choose to return to a film based system. Only one surgeon surveyed prefers film to PACS stating that this is because he is more accustomed to film, having used it over a "long career." All those surveyed stated that their job is either made easier or not affected by 
PACS. No respondents stated that PACS makes their job more difficult.

\section{DISCUSSION}

Satisfying the needs of surgeons and their staff is a particular challenge for a hospital-wide PACS. It is our experience that surgeons in general have less tolerance than other physicians for any factor that might slow or disrupt their work. Radically modifying an element as critical as imaging by switching from film to PACS might be expected to meet with a lack of enthusiasm. In fact, a high level of overall acceptance of the BVAMC PACS by surgeons and staff was evident in interviews and direct observation. Although not without reservations about the system, surgeons and their staff have successfully adapted to a filmless environment and none surveyed would change back to a film-based system if given the opportunity.

The BVAMC PACS was designed and installed before the 1993 hospital opening. Attention was directed towards providing a reliable, integrated hospital-wide system with rapid, convenient access to high quality diagnostic image information. The large-scale and comprehensive nature of the project was rather unprecedented and therefore it was difficult to anticipate many of the specific requirements of different hospital sites and personnel. In the $2 \frac{1}{2}$ years since the PACS became operational, lessons learned from hands-on experience of radiologists, nonradiologist physicians, and other hospital staff have resulted in numerous modifications to the system design and operational protocols. Unfortunately, many suggestions for design improvement have been made which may be difficult to implement retrospectively. However, these suggestions may be of value for others planning a PACS.

On one level, this article presents the results of an analysis of the ability of the BVAMC PACS to fulfill the requirements of surgeons and their staff in the operating room and surgical outpatient clinic. It describes how PACS is used in the surgical arena, areas of satisfaction and dissatisfaction, and suggestions for improvement. On another level, it provides examples of the pitfalls that may arise when implementing a PACS that is designed to both serve the hospital as a whole as well as the specific, widely varying populations that use imaging. Small-scale, specific function PAC systems such as the dedicated ICU PACS described by Tucker et al ${ }^{13}$ and the orthopedic outpatient clinic PACS described by Yamamoto and Kaneda ${ }^{14}$ have been successfully designed for particular clinical areas. The challenge presented to a hospitalwide PACS is to provide the same site-specific functionality to a variety of users in a number of different locations throughout the hospital while maintaining a high level of overall utility. This can be accomplished by constantly monitoring the experience of the different PACS users and incorporating modifications based on their needs. It would be advantageous to involve the nonradiologist PACS users in the original design process because the requirements of various hospital environments for workstation component location and ergonomics may not be well understood by those in the radiology department who may be guiding implementation. ${ }^{15}$ Potential problems and their solutions may be more apparent to those using the technology rather than radiologists or others designing the PACS. ${ }^{16}$

Information from this type of experiential study in which a substantial proportion of the data is necessarily anecdotal is difficult to quantify statistically. However, the individual issues and suggestions elicited may assist others considering a large-scale PACS. As we continue to study the use of PACS by surgeons and their staffs in the OR and clinic, statistical data will be gathered to quantify many of the impressions formed from interviews and observations thus far.

In conclusion, PACS implementation has been successful in the OR and surgical outpatient clinic environments at the BVAMC. The ability of PACS to respond to specific needs of different end-users is essential to the success of a hospital-wide PACS. This suggests that they should be involved early in the design and implementation process. Continued monitoring of system use may result in further "fine tuning" of hardware, software, and imaging protocols in the different areas of the hospital. The radiologist, by virtue of his or her expertise in image quality and management, should work closely with clinical colleagues in the design and evolution of the PACS to optimize its effectiveness in providing patient care. 


\section{REFERENCES}

1. Honeyman JC, Frost MM, Huda W, et al: Picture archiving and communication systems (PACS). Curr Probl Diagn Radiol 23:101-158, 1994

2. Mosser H, Urban M, Hruby W: Filmless digital radiology-feasibility and 20 month experience in clinical routine. Med Inf 19:149-159, 1994

3. Pomerantz SM, Siegel EL, Pickar ER, et al: PACS in the Operating Room: Experience at the Baltimore VA Medical Center Proceedings of the Fourth International Image Management and Communications Symposium (IMAC 95). IEEE Computer Society Press (in press)

4. Siegel EL: Impact of filmless radiology on the Baltimore VA Medical Center. Proceedings of the 1995 Military Telemedicine Symposium, IEEE Computer Society Press (in press)

5. Siegel EL: Plunging into PACS. Diagn Imaging 15:6971,1993

6. Siegel EL, Diaconis JD, Pomerantz SM, et al: Making filmless radiology work. J Digit Imaging 8:151-155, 1995

7. Breant CM, Taira RK, Huang HK: Integration of a voice processor machine in a PACS. Comput Med Imaging Graph 17:13-19, 1993

8. Umeda T, Inamura K, Inamoto K, et al: Development and evaluation of oral reporting system for PACS. Comput Methods Programs Biomed 43:115-123, 1994

9. Strickland NH, Allison DJ: Default display arrange- ments of images on PACS monitors. Br Radiol 68:252-260, 1995

10. Alter AJ, Kargas SA, Cameron JR, et al: The influence of ambient and viewbox light upon visual detection of low contrast targets in a radiograph. Invest Radiol 17:402-406, 1982

11. Rogers DC, Johnston RE: Effect of ambient light on electronically displayed medical images as measured by luminance-discrimination thresholds. J Opt Soc Am [A] 4:976-983, 1987

12. Kuroda C, Yoshioka H, Kadota T, et al: Small PACS for digital medical images - reliability and security in a clinical setting. Comput Methods Programs Biomed 43:101106, 1994

13. Tucker DM, Barnes GT, Koehler RE: Picture archiving communication systems in the intensive care unit. Radiology 196:297-304, 1995

14. Yamamoto I, Kaneda $\mathrm{K}$ : The practical use and evaluation of picture archiving and communication system in the department of orthopaedic surgery. J Digit Imaging 4:25-27, 1991

15. Horii SC: Electronic imaging workstations: Ergonomic issues and the user interface. Radiographics 12:773787,1992

16. Gur D, Fuhrman CR, Thaete FL: Requirements for PACS: Users' perspective. Radiographics 13:457-460, 1993 